\title{
Optimización del tiempo de barrenado en perfiles de aluminio
}

\section{Optimization of the drilling time in aluminum profiles}

\author{
SANTILLAN-VALDELAMAR, Maria Guadalupe †*, DIMAS-DÍAZ, Francelin, SERRANO- \\ GONZÁLEZ, Sergio y RAMOS-HERNÁNDEZ, Cristopher Antonio
}

Instituto Tecnológico Superior del Occidente del Estado de Hidalgo. División de Ingeniería Industrial.

ID $1^{\text {er }}$ Autor: Maria Guadalupe, Santillan-Valdelamar / ORC ID: 0000-0002-3789-9983, CVU CONACYT ID: 1004079

ID 1 ${ }^{\text {er }}$ Coautor: Francelin, Dimas-Díaz / ORC ID: 0000-0002-9414-5424, CVU CONACYT ID: 1019258

ID $2^{\text {do }}$ Coautor: Sergio, Serrano-González / ORC ID: 0000-0003-0252-1259, CVU CONACYT ID: 1004108

ID $3^{\text {er }}$ Coautor: Cristopher Antonio, Ramos Hernández / ORC ID: 0000-0001-6118-9263, CVU CONACYT ID: 1017219

DOI: $10.35429 / J T D .2019 .11 .3 .14 .21$

Recibido: 10 de Julio, 2018; Aceptado 02 de Septiembre, 2018

\section{Resumen}

La rama manufacturera de ventanas de aluminio línea nacional es un área que hoy en día los procesos convencionales son deficientes y con errores al momento de ejecutarse haciendo que el fabricante pierda tiempo, por lo que surge la necesidad de mejorar el proceso de barrenado en los perfiles de aluminio línea nacional para el ensamble de ventanas. El objetivo de este trabajo es construir un troquel para optimizar tiempos y maximizar productividad en el barrenado de perfiles de aluminio línea nacional por medio de un estudio de tiempos, el diseño se realizó utilizando la metodología de Bruno Munari. Con la construcción del troquel para perfiles de aluminio línea nacional se logró la disminución del tiempo de barrenado en promedio de 1.7 minutos a 0.07 minutos que representa la optimización del tiempo en $95.88 \%$ mientras que para ensamblar una ventana se redujo el tiempo de 3.5 minutos a 0.14 minutos lo que representa la maximización de la producción en $96 \%$.

Optimización, Productividad, Estudio de tiempos

\begin{abstract}
The manufacturing branch of aluminum windows national line is an area that nowadays the conventional processes are deficient and with errors at the time of execution causing the manufacturer to lose time, so the need arises to improve the process of drilling in the profiles of aluminum national line for the assembly of windows. The objective of this work is to build a die to optimize times and maximize productivity in the drilling of aluminum profiles national line by means of a study of times, the design was made using the methodology of Bruno Munari. With the construction of the die for aluminum profiles national line was achieved the reduction of the time of drilling on average from 1.7 minutes to 0.07 minutes that represents the optimization of time in $95.88 \%$ while to assemble a window the time of 3.5 minutes was reduced to 0.14 minutes what represents the maximization of the production in $96 \%$.
\end{abstract}

Optimization, Productivity, Study of times

\footnotetext{
* Correspondencia del Autor (Correo electrónico: msantillan@itsoeh.edu.mx)

$\uparrow$ Investigador contribuyendo como primer autor.
} 


\section{Introducción}

En el entorno actual, el área manufacturera está obligada a desarrollar recursos humanos, sistemas de información y capacidades tecnológicas acorde con los nuevos desafíos. Esto implica la renovación y ampliación de procesos, productos y servicios, cambios en la organización, gestión y en la calificación del recurso humano.

En la actualidad no existe alguna máquina o dispositivo que realice el barrenado para perfiles de aluminio línea nacional que son utilizados para el ensamblado de ventanas. El barrenado de perfiles de aluminio línea nacional para ensamble de ventanas en la forma convencional no es uniforme ni limpio, puesto que el operario al momento de barrenar, la broca tiende a barrerse y rayar el perfil provocando pérdidas al fabricante en tiempo, dinero y desperdicio de material. Por lo anterior surge la necesidad de buscar un proceso de barrenado en los perfiles de aluminio línea nacional para el ensamble de ventanas a través del diseño y la construcción de un troquel para perforarlos.

Con el diseño y construcción del troquel para perfiles de aluminio línea nacional se pretende optimizar el tiempo de barrenado y maximizar la productividad.

Se presentan los fundamentos teóricos en este trabajo mencionando las características más importantes de la productividad en las empresas, el estudio de tiempos y movimientos como técnica para incrementar la productividad. Además del software Solidworks como herramienta de diseño para el troquel requerido. Se utilizó la metodología de Bruno Munari para el diseño del troquel comenzando con la definición y elementos del problema en donde se indica el tiempo utilzado para el perforado de manera convencional; la recopilación y análisis de datos donde se toman los tiempos en el perforado; en creatividad se presenta el boceto final del diseño; en materiales, tecnología y experimentación se justifica el material que se utilizará para la construcción, en modelos se presenta el diseño final ensamblado en su totalidad; en la sección de verficación se presenta el prototipo del troquel detallando la funcionalidad de cada componente así como la medición de tiempos para una muestra de 6 ventanas en el perforado de perfiles de aluminio.
En la sección de resultados se presenta la optimización del tiempo en $95.88 \%$, el índice de productividad y la maximización de la producción en $96 \%$.

En la sección de conclusiones se menciona el logro del objetivo con el prototipo para la perforación de perfiles de aluminio línea nacional en función de la problemática de la empresa.

\section{Fundamentos Teóricos}

En los últimos años el avance de la ciencia y tecnología está transformando factores como la competitividad, la calidad, el tiempo, la flexibilidad de los procesos, el valor agregado, disponibilidad de equipos y tecnología. Rojas \& Salas, et al (2006).

La productividad es un indicador relativo que mide la capacidad de un factor productivo, o varios, para crear determinados bienes, por lo que al incrementarla se logran mejores resultados, considerando los recursos empleados para generarlos.

La importancia de la productividad radica en el uso como indicador para medir la situación real de la economía de un país, de una industria o de la gestión empresarial.

A nivel macroeconómico, la productividad incide en numerosos fenómenos económicos y sociales, tales como: crecimiento económico, control de la inflación, empleodesempleo, entre otros.

La productividad se calcula como el cociente entre producción e insumos que debe ser mayor o igual a la unidad. Miranda \&Toirac (2010).

La medición de la productividad es una forma de evaluar la capacidad de un país para mejorar el estándar de vida de su población. Solo mediante el incremento de la productividad puede mejorarse el estándar de vida. Render \& Heizer (2014).

El estudio de tiempos y movimientos es una técnica de la ingeniería industrial que busca aumentar la productividad de las organizaciones, eliminando en forma sistemática las operaciones que no agregan valor al proceso y se constituye en la base para la estandarización de los tiempos de operación.

SANTILLAN-VALDELAMAR, Maria Guadalupe, DIMAS-DÍAZ Francelin, SERRANO-GONZÁLEZ, Sergio y RAMOS-HERNÁNDEZ, Cristopher Antonio. Optimización del tiempo de barrenado en perfiles de aluminio. Revista del Desarrollo Tecnológico. 2019 
El estudio de tiempos y movimientos es una técnica que nos permite identificar fuentes de ineficiencia y desperdicios con el fin de corregir, estandarizar y medir para incrementar la productividad y eficiencia como refieren Palacios (2016) y Tejada et al. (2017). Cruzado (2018).

El diseño en ingeniería es un proceso para resolver problemas en los que se utilizan conocimientos, recursos y productos existentes para crear bienes y procesos nuevos. Bertoline \& Wiebe (1999).

Solidworks es una solución de diseño tridimensional completa que integra un gran número de funciones avanzadas para facilitar el modelado de sólidos en 3D y superficies avanzadas, crear grandes ensambles, generar planos, así como otras funcionalidades que permiten validar, gestionar y comunicar proyectos de forma rápida, precisa y fiable. Gómez S. (2014).

La aplicación del software CAD en la ingeniería abarca la elaboración de cuadros sinópticos, diagramas de diversos tipos, gráficos estadísticos, representación normalizada de piezas para su diseño y fabricación, representación tridimensional de modelos dinámicos en multimedia, análisis con elementos finitos, aplicaciones en realidad virtual, robótica, etc.

El CAD es una técnica de análisis, una manera de crear un modelo del comportamiento de un producto antes de que se haya construido. Rojas \& Rojas (2006)

Los aceros al carbono se fabrican más que cualquier otro acero usados en la construcción, en el campo agroindustrial, aeroespacial, construcción mecánica, industria alimenticia, etc.

La norma SAE trabaja con un sistema que clasifica los aceros en 4 dígitos. El primero especifica la aleación principal, el segundo indica el porcentaje aproximado del elemento principal y los dos últimos dígitos indican la cantidad de carbono en la aleación.

El acero de medio carbono SAE 1018 es usado para fabricación de piezas estructurales, piezas de maquinaria y herramientas.
El porcentaje de carbono de estos aceros va de $0,35 \%$ a $0,40 \%$, tienen una resistencia mecánica de $55 \mathrm{a} 70 \mathrm{~kg} / \mathrm{mm}^{2}$ y una dureza de 180 HB. Con el templado pueden alcanzar una dureza de $90 \mathrm{~kg} / \mathrm{mm}^{2}$. Generalmente se usan en ejes y elementos de máquinas, piezas resistentes, pernos, tornillos, herrajes etc. AHMSA (2011).

\section{Metodología}

En la figura 1 se presenta la metodología proyectual de Bruno Munari que se aplica en el desarrollo del presente trabajo.

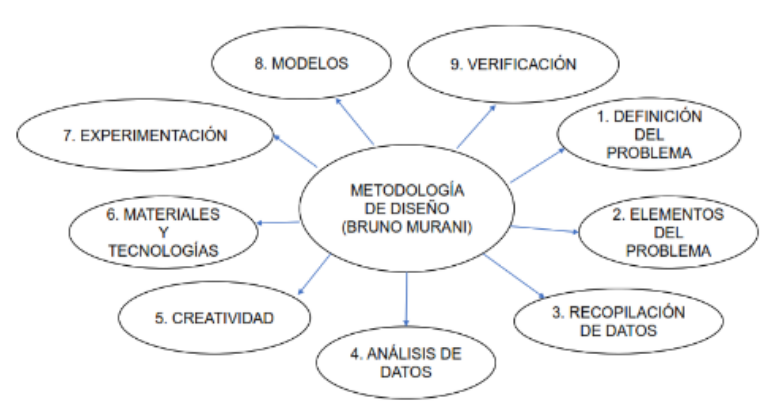

Figura 1 Metodología de Bruno Murani Fuente: Elaboración Propia

Los pasos a llevar a cabo son:
a) Definición del problema
b) Elementos del problema
c) Recopilación de datos
d) Análisis de datos
e) Creatividad
f) Materiales y tecnologías
g) Experimentación
h) Modelos
i) Verificación

\section{Definición y elementos del problema}

La idea de diseñar un troquel para perfiles de aluminio línea nacional surge de la necesidad de mejorar el proceso de perforado en la empresa llamada Vidriera "BAHER" ubicada en la colonia Álamos del municipio de Chilcuautla, Hidalgo.

Para el ensamblado de los perfiles de aluminio se requieren doce barrenos por ventana a ensamblar en sus dos perfiles laterales.

El ensamble de una ventana en forma convencional consta de cuatro perfiles de aluminio: 

a) El perfil superior llamado cabezal.
b) Los perfiles laterales (traslape y cerco).
c) El perfil inferior llamado zoclo.

Los perfiles que se perforan son los laterales (traslape y cerco).

Para ensamblar el cabezal con los dos perfiles laterales se require:

a) Medir en los perfiles laterales $2.2 \mathrm{~cm} \mathrm{de}$ un extremo hacia lo largo y centrarlo

b) Perforar el perfil con una broca de 4.76 $\mathrm{mm}(3 / 16$ in)

c) Barrenar en el mismo punto de un lado con una broca de $9.52 \mathrm{~mm}$ (3/8 in).

El perforar tres veces y con esas medidas es porque se introduce un tornillo por los barrenos de los perfiles laterales para después atornillar con el cabezal o zoclo en la guía que tienen para ensamblarse.

El proceso para ensamblar el zoclo es el mismo que el del cabezal solo que la medida es de $4.4 \mathrm{~cm}$.

Este proceso de ensamblado de ventanas se convierte en una actividad morosa ya que se hacen mediciones para cada barreno, se cambian las brocas constantemente, la broca tiende a barrerse y rayar el material obteniéndose un perforado no uniforme en los perfiles afectando a la empresa en el incremento de costos, mano de obra, tiempo y desperdicio de material.

\section{Recopilación y análisis de datos}

Se registraron los tiempos que lleva el proceso de barrenado en los perfiles de aluminio en la forma convencional con el armado de 6 ventanas, cada una requiere dos perfiles de aluminio, teniendo un total 12 perfiles de aluminio a perforar.

En la Tabla 1 se muestra que el tiempo total de barrenado para los 12 perfiles de aluminio es de 20.7 minutos, que en promedio el tiempo por perfil es de 1.725 minutos; mientras que en promedio por ventana el tiempo de perforar es de 3.45 minutos (se consideran 2 perfiles por ventana).

\begin{tabular}{|c|c|c|c|}
\hline $\begin{array}{c}\text { No. de } \\
\text { ventanas }\end{array}$ & $\begin{array}{l}\text { No. de perfiles } \\
\text { de aluminio } \\
\text { perforados }\end{array}$ & $\begin{array}{c}\text { Tiempo de } \\
\text { barrenado por } \\
\text { perfil (min) }\end{array}$ & $\begin{array}{c}\text { Tiempo de } \\
\text { barrenado por } \\
\text { ventana (min) }\end{array}$ \\
\hline \multirow{2}{*}{1} & 1 & 1.6 & \multirow{2}{*}{3.4} \\
\hline & 2 & 1.8 & \\
\hline \multirow{2}{*}{2} & 3 & 1.8 & \multirow{2}{*}{3.5} \\
\hline & 4 & 1.7 & \\
\hline \multirow{2}{*}{3} & 5 & 1.9 & \multirow{2}{*}{3.6} \\
\hline & 6 & 1.7 & \\
\hline \multirow{2}{*}{4} & 7 & 1.6 & \multirow{2}{*}{3.3} \\
\hline & 8 & 1.7 & \\
\hline \multirow{2}{*}{5} & 9 & 1.8 & \multirow{2}{*}{3.5} \\
\hline & 10 & 1.7 & \\
\hline \multirow{2}{*}{6} & 11 & 1.6 & \multirow{2}{*}{3.4} \\
\hline & 12 & 1.8 & \\
\hline \multicolumn{2}{|c|}{ Tiempo total (min) } & 20.7 & 20.7 \\
\hline \multicolumn{2}{|c|}{ Tiempo promedio (min) } & 1.7 & 3.5 \\
\hline
\end{tabular}

Tabla 1 Tiempo de barrenado Fuente: Elaboración Propia

En la tabla 2 se registra la cantidad de barrenos que se requieren para el armado de 6 ventanas (12 perfiles de aluminio perforados).

\begin{tabular}{|c|c|c|c|}
\hline $\begin{array}{c}\text { No. de } \\
\text { ventanas }\end{array}$ & $\begin{array}{c}\text { No. de perfiles } \\
\text { de aluminio } \\
\text { perforados }\end{array}$ & $\begin{array}{c}\text { Tiempo de } \\
\text { barrenado por } \\
\text { perfil (min) }\end{array}$ & $\begin{array}{c}\text { Cantidad de } \\
\text { barrenos }\end{array}$ \\
\hline \multirow{2}{*}{1} & 1 & 1.6 & 6 \\
\hline & 2 & 1.8 & 6 \\
\hline \multirow{2}{*}{2} & 3 & 1.8 & 6 \\
\hline & 4 & 1.7 & 6 \\
\hline \multirow{2}{*}{3} & 5 & 1.9 & 6 \\
\hline & 6 & 1.7 & 6 \\
\hline \multirow{2}{*}{4} & 7 & 1.6 & 6 \\
\hline & 8 & 1.7 & 6 \\
\hline \multirow{2}{*}{5} & 9 & 1.8 & 6 \\
\hline & 10 & 1.7 & 6 \\
\hline \multirow{2}{*}{6} & 11 & 1.6 & 6 \\
\hline & 12 & 1.8 & 6 \\
\hline \multicolumn{3}{|c|}{ Total de barrenos $=$} & 72 \\
\hline
\end{tabular}

Tabla 2 Registro de barrenos

Fuente: Elaboración Propia

\section{Creatividad}

Después de estudiar la forma convencional de hacer barrenos en los perfiles de aluminio se realizaron varios bocetos, en la figura 2 se muestra boceto final.

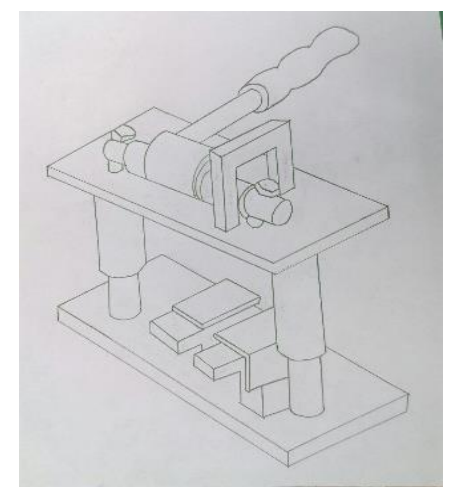

Figura 2 Boceto final

SANTILLAN-VALDELAMAR, Maria Guadalupe, DIMAS-DÍAZ, Francelin, SERRANO-GONZÁLEZ, Sergio y RAMOS-HERNÁNDEZ, Cristopher Antonio. Optimización del tiempo de barrenado en perfiles de aluminio. Revista del Desarrollo Tecnológico. 2019 


\section{Materiales, tecnología y experimentación}

Se investigaron los tipos de aceros que existen en el mercado para seleccionar el acero con el cual se fabricará el troquel, se determinó que el acero al carbón 1018 es la mejor opción ya que es un producto útil debido a sus características típicas de resistencia mecánica y ductilidad, excelente soldabilidad y mejor maquinabilidad que la mayoría de los aceros al carbón. Además al ser sometido a cementado para tener una superficie dura pero un centro de gran tenacidad y por su ductilidad se usa en procesos de recalcado, doblado o estampado.

5. Modelos

El diseño de la herramienta consta de cinco sub-ensambles: 1. Maneral, 2. Corte, 3. Base, 4. Soporte de corte izquierdo y 5. Soporte de corte derecho. El ensamble general se muestra en la figura 3.

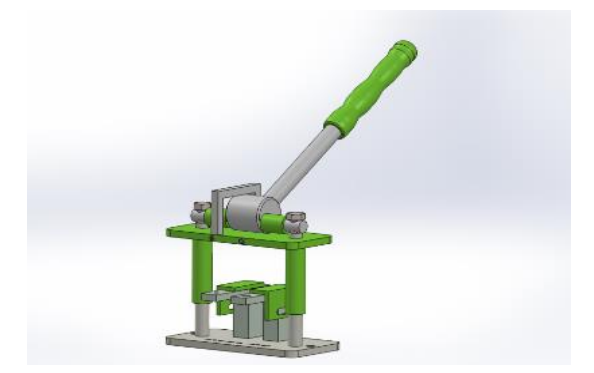

Figura 3 Ensamble final del diseño Fuente: Elaboración Propia

\section{Verificación}

Todos los diseños son elaborados por medio del software Solidworks para obtener un diseño preciso del troquel y evitar gastos extras en su fabricación. Después del diseño del prototipo, en la figura 4 se presenta la construcción del troquel para perfiles de aluminio línea nacional. Las dimensiones son de 15 centímetros de altura por 20 centímetros de ancho.

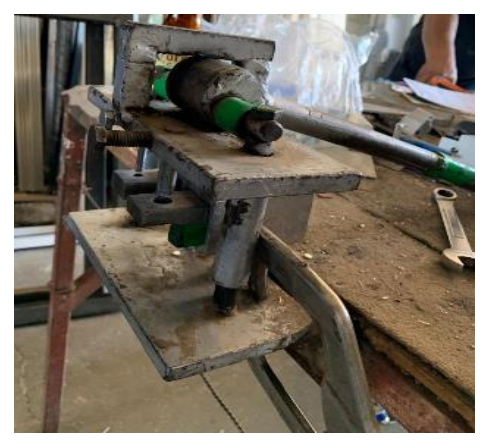

Figura 4 Prototipo

Fuente: Elaboración Propia
En la Figura 5 se presenta la parte superior del troquel en la que se observa el sistema manual para realizar el perforado junto con las brocas de $4.76 \mathrm{~mm}$ (3/16 in) y $9.52 \mathrm{~mm}$ (3/8 in). La pieza base para ejercer la presión requerida es el maneral, el operario se coloca frente al troquel, con una mano toma el mango del maneral y lo mueve en dirección de su pecho para que el excéntrico gire. Al momento de girar el concéntrico se produce un empuje a la placa superior de acero con un movimiento hacia abajo guiado por dos puntales que están soldados a la placa inferior del troquel generándose la perforación.

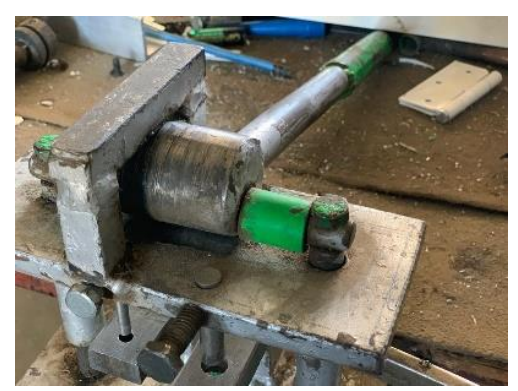

Figura 5 Parte superior del troquel

Fuente: Elaboración Propia

También se observa que el concéntrico cuenta con una guía y dos espaciadores de cada lado para mantener el concéntrico en un solo punto y evitar un desplazamiento. Se debe mantener lubricado el excéntrico para evitar una fricción excesiva o desgaste al momento de girar el excéntrico sobre la placa superior del troquel lo que permite mantener un giro suave y cómodo para el operario.

La Figura 6 muestra los dos soportes para perfiles de aluminio con diámetros de 4.76 $\mathrm{mm}(3 / 16$ in) y $9.52 \mathrm{~mm}(3 / 8 \mathrm{in})$ respectivamente para realizar la perforación al momento de introducir los perfiles de aluminio. Los soportes cuentan con dos topes (derecho e izquierdo) que permiten introducir el perfil eliminando el margen de error que existe en la forma convencional respecto a la posición de cada barreno.

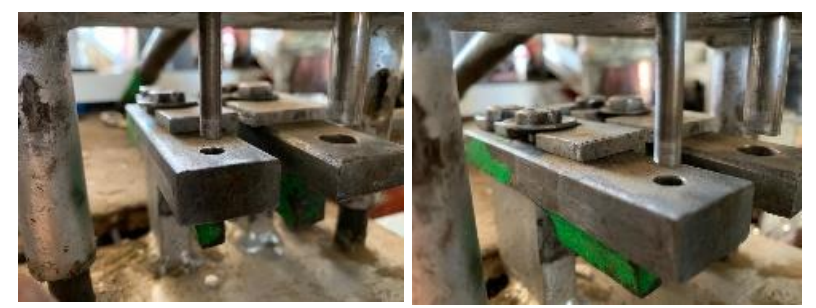

Figura 6 Soportes de perfiles de aluminio Fuente: Elaboración Propia 
El tope se utiliza al perforar el lado superior de los dos perfiles laterales de la ventana a ensamblar que se unirán al cabezal colocando el perfil a 2.2 centímetros. Para perforar el lado inferior de los dos perfiles laterales de la ventana a ensamblar es necesario quitar los topes para colocar el perfil a 4.4 centímetros.

En la punta de los dos soportes para introducir los perfiles de aluminio cuentan con un barreno con el diámetro respectivo de las brocas asignadas, cada barreno tiene una tolerancia de $0.799 \mathrm{~mm}$ para evitar fricción entre la broca y el soporte con el fin de disminuir un desgaste o deformidad entre ellos y mantener un corte uniforme.

Los soportes tienen además huecos de escape para el material cortado, son barrenos con diámetro de $12.7 \mathrm{~mm}$ (1/2in) con una profundidad de $5 \mathrm{~mm}$ para ambos soportes con el fin de liberar los residuos del material cortado ya que cuando se perfora el perfil las hojuelas de aluminio tienden a estancarse dentro del barreno al hacer varias perforaciones.

En la figura 7 se presenta el portabrocas que se encuentra en la base superior del troquel, las cuales son de punta de carburo, ideales para el corte de aluminio.

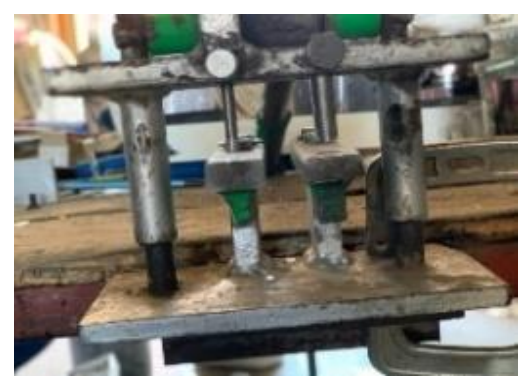

Figura 7 Porta brocas

Fuente: Elaboración Propia

El troquel para perfiles de aluminio cuenta con un sistema de reemplazo de brocas debido al uso constante y desgaste.

Para realizar el cambio las brocas se introducen por debajo de la base superior del troquel, se fijan con tornillos de presión que se introducen por la parte frontal de la base superior del troquel, la broca del lado derecho de diámetro $9.52 \mathrm{~mm}(3 / 8 \mathrm{in})$ y la broca del lado izquierdo de diámetro $4.76 \mathrm{~mm}$ (3/16 in) son fijadas con tornillos de presión de $6.35 \mathrm{~mm}(1 / 4$ in) de diámetro.
La base inferior del troquel para perfiles de aluminio se observa en la figura 8 , que se compone de los soportes para introducir los perfiles de aluminio y los dos puntales para guiar el movimiento del perforado.

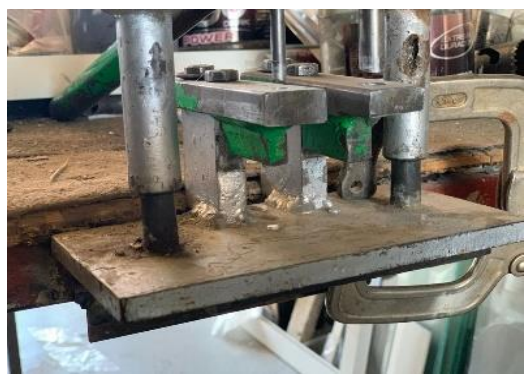

Figura 8 Base inferior

Fuente: Elaboración Propia

Con el troquel para perfiles de aluminio se obtiene una misma posición del barreno puesto que cuenta con las medidas requeridas al introducir el perfil de aluminio para ser perforado y con ello evitar el margen de error que existe en la forma convencional al medir manualmente, además de lograr un perforado uniforme y limpio en los perfiles de aluminio como se muestra en la figura 9.

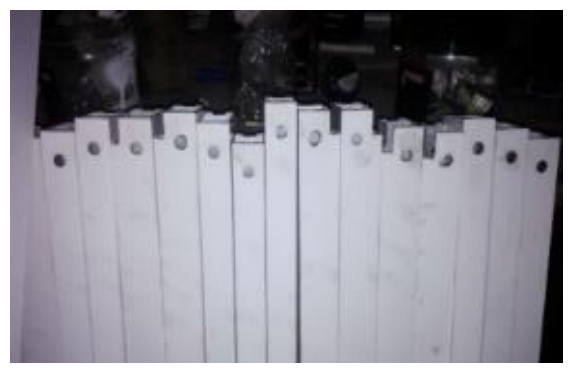

Figura 9 Barrenos

Fuente: Elaboración Propia

Una vez elaborado el prototipo se llevó a cabo la toma de tiempos para realizar el comparativo del método convencional con el prototipo.

Se muestra en la Tabla 3 el tiempo total de perforado para el armado de 6 ventanas, con un total de 12 perfiles de aluminio a perforar en un tiempo promedio de 0.82 minutos; el tiempo promedio de perforado por perfil de aluminio fue de 0.7 minutos y el tiempo promedio por ventana fue de 0.14 minutos. 


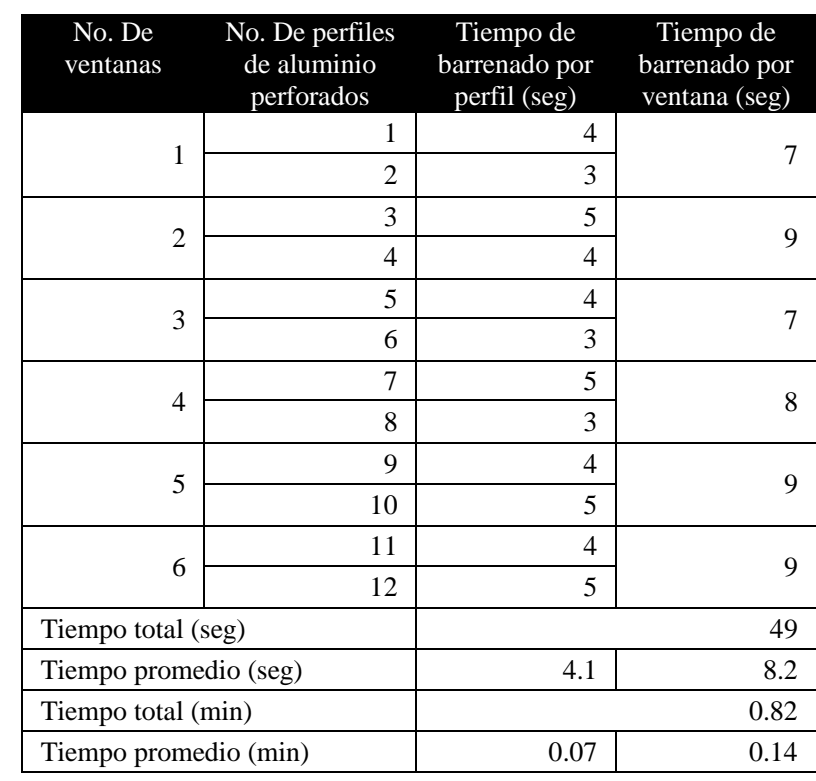

Tabla 3 Registro de tiempos con troquel Fuente: Elaboración Propia

En la Tabla 4 se muestra el comparativo de registro de tiempos mostrando la eficiencia del troquel para el armado de 6 ventanas y el tiempo promedio de perforado por perfil de aluminio línea nacional. Se logra reducir el tiempo total de perforado, de 20.7 minutos a 0.8 minutos. El tiempo promedio de perforado por perfil de aluminio se reduce de 1.7 minutos a 0.07 minutos y finalmente se logra disminuir el tiempo para ensamblar una ventana de 3.5 minutos a 0.14 minutos.

Por lo anterior el troquel para perfiles de aluminio línea nacional logró minimizar el tiempo de barrenado en los perfiles en $95.88 \%$ mientras que para el ensamblado de las ventanas se logró maximizar la producción en $96 \%$.

\begin{tabular}{|c|c|c|c|c|c|}
\hline \multirow[b]{2}{*}{$\begin{array}{l}\text { No. De } \\
\text { Ventana } \\
\text { s }\end{array}$} & \multirow[b]{2}{*}{$\begin{array}{l}\text { No. De } \\
\text { perfiles de } \\
\text { aluminio } \\
\text { perforado } \\
\text { s }\end{array}$} & \multicolumn{2}{|c|}{$\begin{array}{c}\text { De forma } \\
\text { convencional }\end{array}$} & \multicolumn{2}{|c|}{$\begin{array}{l}\text { Con el troquel para } \\
\text { perfiles de } \\
\text { aluminio }\end{array}$} \\
\hline & & $\begin{array}{l}\text { Tiempo de } \\
\text { barrenado } \\
\text { por perfil } \\
\text { (min) }\end{array}$ & $\begin{array}{l}\text { Tiempo de } \\
\text { barrenado } \\
\text { por } \\
\text { ventana } \\
\text { (min) }\end{array}$ & $\begin{array}{l}\text { Tiempo } \\
\quad \text { de } \\
\text { barrenado } \\
\text { por perfil } \\
\text { (min) }\end{array}$ & $\begin{array}{l}\text { Tiempo } \\
\text { de } \\
\text { barrenado } \\
\text { por } \\
\text { ventana } \\
\text { (min) }\end{array}$ \\
\hline \multirow{2}{*}{1} & 1 & 1.6 & \multirow{2}{*}{3.4} & 0.07 & \multirow{2}{*}{0.12} \\
\hline & 2 & 1.8 & & 0.05 & \\
\hline \multirow{2}{*}{2} & 3 & 1.8 & \multirow{2}{*}{3.5} & 0.08 & \multirow{2}{*}{0.15} \\
\hline & 4 & 1.7 & & 0.07 & \\
\hline \multirow{2}{*}{3} & 5 & 1.9 & \multirow{2}{*}{3.6} & 0.07 & \multirow{2}{*}{0.12} \\
\hline & 6 & 1.7 & & 0.05 & \\
\hline \multirow{2}{*}{4} & 7 & 1.6 & \multirow{2}{*}{3.3} & 0.08 & \multirow{2}{*}{0.13} \\
\hline & 8 & 1.7 & & 0.05 & \\
\hline \multirow{2}{*}{5} & 9 & 1.8 & \multirow{2}{*}{3.5} & 0.07 & \multirow{2}{*}{0.15} \\
\hline & 10 & 1.7 & & 0.08 & \\
\hline \multirow{2}{*}{6} & 11 & 1.6 & \multirow{2}{*}{3.4} & 0.07 & \multirow{2}{*}{0.15} \\
\hline & 12 & 1.8 & & 0.08 & \\
\hline \multirow{2}{*}{\multicolumn{2}{|c|}{$\begin{array}{r}\text { Tiempo total (min) } \\
\text { Tiempo promedio } \\
(\text { min) }\end{array}$}} & \multicolumn{2}{|r|}{20.7} & \multicolumn{2}{|r|}{0.8} \\
\hline & & 1.7 & 3.5 & 0.07 & 0.14 \\
\hline
\end{tabular}

Tabla 4 Tabla comparativa de registro de tiempos

\section{Maximización de la producción}

De acuerdo a las necesidades de producción de la empresa se requerían producir 6 ventanas, motivo por el cual se tomó ese número como la muestra para realizar las pruebas del troquel de perfiles de aluminio línea nacional.

Se tomó la muestra de 6 ventanas a ensamblar con 12 perfiles de aluminio a perforar. Se observa que la forma convencional necesita un tiempo de 20.7 minutos para realizar 72 barrenos, en comparación al troquel para perfiles de aluminio requiere un tiempo de 0.82 minutos para realizar 72 barrenos. En la tabla 5 se muestra la tabla comparativa de la cantidad de barrenos.

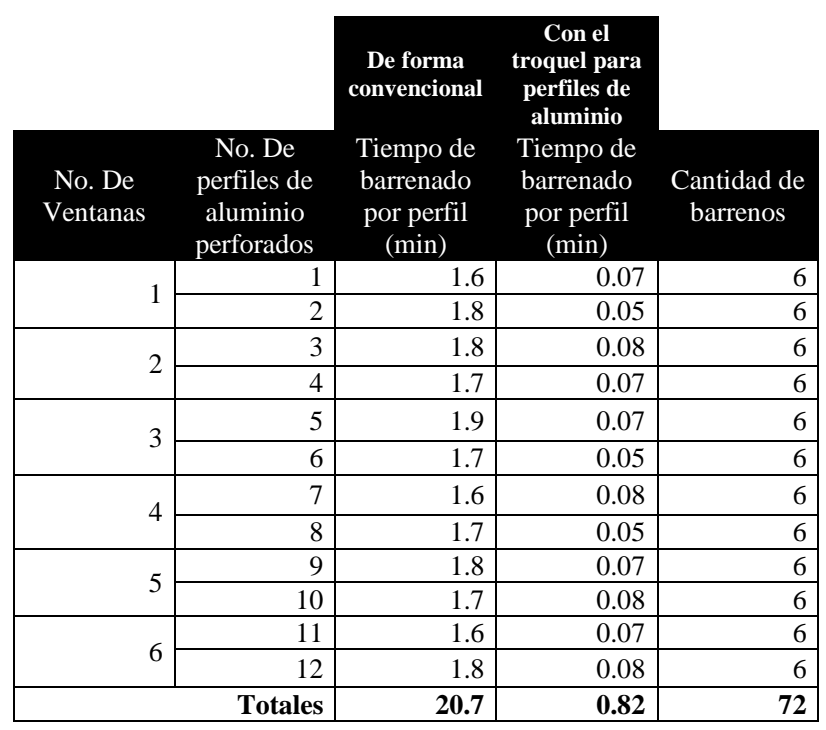

Tabla 5 Tabla comparativa de cantidad de barrenos Fuente: Elaboración Propia

\section{Índice de productividad}

Con los resultados obtenidos de la Tabla 5 se calcula el índice de productividad de cada método con la fórmula (1).

$$
\text { índice de productividad }=\frac{\text { producción }}{\text { consumo }}
$$

Con el método convencional se obtiene un índice de productividad de 3.47 , lo que significa que por cada minuto se hacen casi 4 perforaciones.

\section{$\frac{72 \text { barrenos }}{20.7 \text { minutos }}=3.47$}

Con el troquel para perfiles de aluminio línea nacional se obtiene un índice de productividad de 80.87 , lo que significa que por cada minuto se pueden realizar casi 81 barrenos.

SANTILLAN-VALDELAMAR, Maria Guadalupe, DIMAS-DÍAZ, Francelin, SERRANO-GONZÁLEZ, Sergio y RAMOS-HERNÁNDEZ, Cristopher Antonio. Optimización del tiempo de barrenado en perfiles de aluminio. Revista del Desarrollo Tecnológico. 2019 
$\frac{72 \text { barrenos }}{0.82 \text { minutos }}=80.87$

\section{Agradecimientos}

Se agradecen las facilidades y el apoyo otorgados por la empresa Vidrieria "BAHER" para la realización del prototipo y las pruebas requeridas.

\section{Resultados}

Gracias al diseño del troquel para perfiles de aluminio, la herramienta es fácil de usar y práctica para el operario dejando el mantenimiento excesivo con solo mantener lubricado el concéntrico para garantizar el funcionamiento del troquel.

Se logró la reducción de tiempo de barrenado en $95.88 \%$, la maximización en el ensamblado de ventanas en $96 \%$ y el índice de productividad de 80.87 .

\section{Conclusiones}

Con el diseño y la construcción del troquel para perfiles de aluminio línea nacional se logró disminuir el tiempo de perforado, maximizar la producción e incrementar el índice de productividad dando solución a la problemática planteada por la empresa.

\section{Referencias}

Altos Hornos de México (AHMSA). (2011). Manual de especificaciones y garantías AHMSA. Monclova, Coahuila: Impreso en México. Recuperado el 16 de Noviembre de 2018, de www.ahmsa.com

Bertoline G., Wiebe E. (1999). Dibujo en Ingeniería y Comunicación Gráfica. Edit. Mc Graw Hill. México.

Chase, R. B; Jacobs, F. R. (2014). Administración de operaciones. Producción y cadena de suministros. Decimotercera edición. Mc Graw Hill. México.

Cruzado Ruiz, D. Y. (2018) El estudio de tiempos y movimientos en los procesos de producción: una revisión sistemática. Universidad Privada del Norte. Ingenieria Industrial. Cajamarca-Perú.
Gómez S. (2014). SolidWorks Práctico I. Edit Alfaomega. México.

Hernández, S; Fernández, C; Baptista, L. (2014) Metodología de la investigación. $6^{\text {a }}$. Edición. Mc Graw Hill. México.

Jennsen C, Helsel J. (2004). Dibujo y Diseño en Ingeniería. Edit. Mc Graw Hill. México.

Miranda, J; Toirac, L. (2010) Indicadores de productividad para la industria dominicana. Ciencia y Sociedad, vol. XXXV, núm. 2, pp. 235-290. Disponible en: http://www.redalyc.org/articulo.oa?id=8701456 3005

Mott, R; (2006). Diseño de elementos de máquinas. Pearson Educación. México

Munari, B. (1993) ¿Cómo nacen los objetos? México: Ediciones G. Gilli, S.A. de C.V.

Ovalle-Castiblanco, A. M., Cárdenas-Aguirre, D. M. (2016) ¿Qué ha pasado con la aplicación del estudio de tiempos y movimientos en las últimas dos décadas? Revista Ingeniería, Investigación y Desarrollo, Vol. 16 N² 2, pp. 1231, Sogamoso-Boyacá. Colombia.

Render, B; Heizer, J; (2014). Principios de Investigación de operaciones. Novena edición. Pearson Educación. México.

Rojas Lazo, O., \& Rojas Rojas, L. (2014). Diseño asistido por computador. Industrial Data, 9(1), 007-015. DOI: https://doi.org/10.15381/idata.v9i1.5709

Rojas Lazo, O; Salas Bacalla, J; Santos de la Cruz, E; Marín Chávez, P; Mejía Elías, C. (2006)

Enseñanza del diseño asistido por computador en la Facultad de Ingeniería Industrial, UNMSM Industrial Data, vol. 9, núm. 1, pp. 16-22 Disponible en: http://www.redalyc.org/articulo.oa?id=8169010 3

Vilchis (2002). Metodología del Diseño. Editorial Claves Latinoamericanas. México 